$\underline{\text { Reports }}$

\title{
Promising practices for adapting and implementing the WHO Safe Childbirth Checklist: case studies from India and Rwanda
}

\author{
Rose L. Molina ${ }^{1}$ ๑, Anuradha Pichumani ${ }^{2}$, Eugene Tuyishime ${ }^{3}$, Lauren Bobanski ${ }^{4}$, Katherine E. A. Semrau ${ }^{5}$ \\ ${ }^{1}$ Department of Obstetrics \& Gynecology, Beth Israel Deaconess Medical Center; Ariadne Labs | Brigham and Women's Hospital and Harvard T.H. \\ Chan School of Public Health, 2 Obstetrics \& Gynecology, Sree Renga Hospital, ${ }^{3}$ Department of Anesthesia and Pain Management, University of \\ Ottawa, Canada; Department of Anesthesia, Critical Care, and Emergency Medicine, University of Rwanda, ${ }^{4}$ Ariadne Labs | Brigham and Women's \\ Hospital and Harvard T.H. Chan School of Public Health, ${ }^{5}$ Ariadne Labs | Brigham and Women's Hospital and Harvard T.H. Chan School of Public \\ Health; Department of Medicine, Brigham and Women's Hospital; Division of Global Health Equity, Brigham \& Women's Hospital \\ Keywords: safe childbirth checklist, implementation, quality of care, maternal health \\ https://doi.org/10.29392/001c.30751
}

Journal of Global Health Reports

Vol. 5, 2021

\begin{abstract}
Background
The World Health Organization (WHO) Safe Childbirth Checklist (SCC) was published in 2015 as a patient safety tool to improve facility-based childbirth care through boosting adherence with essential practices around the major causes of maternal and newborn morbidity and mortality. We brought together partners who led implementation of the SCC in India and Rwanda to: (i) contextualize the findings from surveys and interviews about SCC adaptation and implementation around the world (data published separately) with our partners' implementation experiences in India and Rwanda, and (ii) identify promising practices for SCC implementation.
\end{abstract}

\begin{abstract}
Methods
We identified two partners-one from Tamil Nadu, India and one from Masaka District, Rwanda-to work together in identifying key promising practices regarding the SCC based on their direct experiences and data we collected from other implementers around the world. From June-September 2020, we held 4 virtual design workshops using brainwriting exercises to explore promising practices for adaptation and implementation of the SCC. We consolidated the implementation experiences in India and Rwanda into the WHO SCC Implementation Guide phases of Engage, Launch, and Support, and included two additional phases: Project Design and Evaluation.
\end{abstract}

\section{Results}

We present two case studies of SCC implementation that demonstrate improved adherence with essential birth practices after implementation of the SCC. Based on the case studies, we developed promising practices according to five implementation stages: Project Design, Engage, Launch, Support, and Evaluation. Clarifying the purpose and users of the tool, applying human-centered design principles, and developing evaluation plans for the specified purpose were some promising practices that emerged.

\section{Conclusions}

Our partnership with direct implementers of the SCC yielded important insights about how to adapt, implement, evaluate, and sustain use of the Checklist. Such partnerships are critical in building an evidence base for promising practices regarding SCC implementation around the world.

The World Health Organization (WHO) Safe Childbirth Checklist (SCC) was published in 2015 as a patient safety tool to improve facility-based childbirth care through boosting adherence with essential practices to prevent the major causes of maternal and newborn morbidity and mortality. ${ }^{1}$ There is increasing evidence that the SCC is a vital tool in reducing perinatal mortality when implemented as part of broader quality improvement initiatives, such as enhanced supply chains in India ${ }^{2}$ and technical training, qual- ity improvement collaboratives, and data quality assurance in Kenya and Uganda. ${ }^{3}$ Over the past 5 years, there has been increasing adaptation and implementation of the SCC in a variety of settings, ${ }^{4}$ including Brazil, ${ }^{5-7}$ Ethiopia, ${ }^{8,9}$ Indonesia, ${ }^{10}$ and Italy. ${ }^{11}$ With this uptake of the SCC, there is an opportunity to identify promising practices regarding SCC adaptation, implementation and evaluation. Contextspecific factors, such as facility infrastructure, supply chains, teamwork culture, and quality improvement capac- 
ity, may have implications for how to best adapt and implement the SCC in a particular site. We brought together partners who led implementation of the SCC in India and Rwanda to integrate lessons learned and identify promising practices for implementers around the world.

\section{METHODS}

Ariadne Labs, a joint center for health system innovation between Brigham and Women's Hospital and Harvard T.H. Chan School of Public Health, was a key partner in the design and evaluation of the BetterBirth Trial, ${ }^{12}$ the largest cluster randomized controlled trial of the SCC to date. The BetterBirth Program team at Ariadne Labs has been dedicated to continuous learning about the SCC to understand its optimal implementation across settings. We identified two partners-one from Tamil Nadu, India and one from Kigali, Rwanda-to work together in identifying key promising practices regarding the SCC based on their direct experiences and data collected from other global implementers. ${ }^{4}$ We selected these partners based on their enthusiasm as local champions of the SCC in their hospitals and their desire to improve and scale-up SCC use.

From June-September 2020, we held 4 virtual design workshops with our partners to accomplish the following aims: 1) contextualize the findings from surveys and interviews about SCC adaptation and implementation around the world ${ }^{4}$ with our partners' implementation experiences in India and Rwanda, 2) identify promising practices for SCC tool adaptation, 3) identify promising practices for SCC implementation, and 4) integrate and finalize promising practices. For the design workshops, we used brainwriting exercises $^{13}$ to facilitate ideation amongst remote participants. The brainwriting exercises were a series of prompts that each participant responded to and built off of each other's ideas in a rotating manner. The order of respondents was randomly assigned to elicit different perspectives. The responses were consolidated and sent to our field partners for review and finalization. The exercises focused on creating recommendations on promising practices for adaptation and implementation of the SCC based on the findings of the surveys and interviews and the experience of our two expert partners as well as the experience with the BetterBirth trial at Ariadne Labs. We consolidated the implementation experiences according to the phases of Engage, Launch, and Support described in the WHO SCC Implementation Guide, which the WHO published along with the SCC in $2015 .{ }^{14}$ We also developed promising practices in two additional phases: Project Design and Evaluation. In this Field Action Report, we summarize the case studies from India and Rwanda and present the promising practices we co-developed based on these experiences.

\section{RESULTS}

\section{CASE STUDY: INDIA}

Implementation context: In early 2020, the WHO SCC was introduced in Sree Renga Hospital, a 47-bed multispeciality, private hospital that serves a rural and semi-urban population in Chengalpattu, Tamil Nadu, India, with a strong focus on adopting and implementing healthcare quality practices. As part of its Continual Quality Improvement initiative, the hospital has implemented the WHO Safe Surgery Checklist since 2010. To raise the standards of childbirth care, the hospital developed the adaptation and implementation of the WHO SCC in partnership with Ariadne Labs in early 2020.

Project design: The project was integrated into the Quality Improvement initiative of the Labour Ward in February 2020. The WHO SCC was reviewed periodically, and feedback from all stakeholders was incorporated. More than 40 new clinical and operational items were introduced into the Checklist to align with local regulatory and statutory requirements.

Engage: The Engage phase included gaining support from the hospital leaders and managers, which facilitated processes and resources to accomplish project goals. A comprehensive project team was assembled, including 3 obstetricians, 2 anesthetists, 2 pediatricians, 8 nurses, 4 nurses aides, and 3 technicians. Feedback from all stakeholders was incorporated and resulted in enhanced motivation and adherence with the project implementation. The project team led by Dr. Anuradha Pichumani conducted a gap assessment to identify additional resources and skills needed for successful implementation.

To address gaps in technical competency around childbirth care, the hospital leadership implemented a set of technical trainings for the staff, including: (i) Labor Ward practices by obstetricians, (ii) Kangaroo Mother Care and newborn resuscitation by pediatricians and anesthetists, and (iii) data collection, analysis, and dissemination of SCC data for the Medical Records Department and Quality Department. External organizations provided additional technical training, including the Manyata initiative to improve competency and confidence in providing safe and respectful maternity care. Topics included management of eclampsia, preterm premature rupture of membranes, and postpartum hemorrhage. The Sree Renga Hospital obtained Manyata certification in October 2020, alongside the implementation of the SCC. Other trainings were focused on infection control practices, communication skills for nurses on the labor ward, and quality standards and audits. Due to the COVID-19 pandemic, some training programs shifted to a virtual platform to comply with social distancing restrictions.

Launch: Sree Renga Hospital held an official launch of the WHO SCC during the hospital-wide monthly committee meeting in February 2020. Hospital leadership participated to increase buy-in from managers and to introduce the SCC team to all other departments of the hospital. Obstetricians discussed the challenges in the implementation process. Coordination of all staff and incorporation of their feedback were priorities to increase accountability in the SCC implementation. The COVID-19 pandemic posed additional challenges due to the rapidly changing hospital protocols to respond to increasing COVID-19 caseloads, which had to be implemented alongside the SCC.

Support: With support of the hospital leadership, the Support phase consisted of continuous coaching and motivation to improve adherence to practices. Initially, in-person coaching took place on a weekly basis for four weeks 
and included live demonstrations on preparing the delivery tray, postpartum hemorrhage training, basic life support training, and newborn resuscitation. There were periodic didactic trainings augmented with in-person skills sessions. During the COVID-19 pandemic, these trainings were moved into the virtual Zoom platform. The SCC implementation team sought constructive feedback from all participants, including obstetricians, and incorporated relevant ideas into the workflow. A nurse champion helped lead the implementation process and assessed gaps in technical skills, particularly for newly hired nurses. After the initial four-week phase, coaching was held monthly and intermittently as new staff members were added to the team. Continual feedback via WhatsApp groups helped with coaching improvements in real time.

Rapid changes in the regulatory protocols for patient admission during the COVID-19 pandemic (imposition of lockdowns, disrupted availability of transportation options and mandatory referral of COVID-positive patients to government-designated facilities for delivery) reduced the number of patients presenting to Sree Renga Hospital for delivery and impacted the implementation of WHO SCC due to changes in clinical workflows and modality of staff trainings and communication. Timely, manual verification of filling out the WHO SCC forms at various pause points was inconvenient as it exposed the Medical Records and Quality department staff to the high-risk areas of the hospital. Digital communication over a WhatsApp group was heavily encouraged to mitigate the challenge without compromising the intent for quality improvement. Despite such challenges during pandemic, the implementation team successfully engaged the stakeholders, launched the initiative, and provided support for continual improvement.

Evaluation: The implementation progress was measured in two different quality improvement cycles: Cycle 1 (March - July 2020) and Cycle 2 (August 2020 - December 2020). In Cycle 1, the aim was to ensure completion of all elements of the WHO SCC at the four pause points. Obstetricians were engaged in this phase and identified opportunities for improvement. Completed checklists were collected by the medical records department. The quality department audited the checklists for completion. Adherence to completion of the essential birth practices improved from $48 \%$ to $77 \%$ by the end of Cycle 1 . In Cycle 2, the aim was to increase adherence to WHO SCC from $77 \%$ to $90 \%$ and ensure timely completion of all elements of the WHO SCC. A WhatsApp group of obstetricians, nurses, and quality department members monitored completed SCC forms that were posted to the group. By the end of Cycle 2, completion of the WHO SCC averaged $94 \%$ with $70 \%$ compliance with timely completion.

The results were discussed among the Medical Records and Quality departments and the staff of the Labour Ward, before being presented in the monthly quality committee meeting. Suitable Corrective Action and Preventive Action plans were recommended and implemented to address challenges that emerged. Recognition and rewards were given for good performance.

Challenges and lessons learned: The perception of the WHO Safe Childbirth Checklist as an additional paperwork burden was a challenge to successful implementation. To make it less burdensome, the SCC was included in the patient medical record as standard documentation. We explained to the team the importance of the SCC in increasing safety and quality of care delivery for patients. Documentation of the partograph was also a challenge initially, which was due to the lack of proper training on its use. We included training on how to use the partograph, which obstetricians provided to all staff members. Ensuring consistent behavior change on sticky practices, such as hand washing, breastfeeding, and skin-to-skin contact, was a challenge that we addressed through inclusion of a local champion to motivate staff members. We also created a WhatsApp group to address concerns about SCC implementation in real time.

Low-cost implementation of the WHO SCC has strengthened the hospital's processes and boosted confidence among staff in delivering safe obstetric care. Handovers amongst staff nurses during shift changes became easier, and errors due to miscommunication were reduced. Despite the challenges to regular processes imposed by the COVID-19 pandemic, sustained implementation and continual improvements were possible with continuous engagement of all stakeholders.

\section{CASE STUDY: RWANDA}

Implementation context: Masaka District Hospital has 35 maternity beds and offers both emergency obstetric care and cesarean deliveries. In 2016, the average hospital length of stay was one day after vaginal delivery and three days after cesarean delivery. Approximately, 500 vaginal deliveries were performed monthly. During the project implementation period, the maternity unit team included 20 midwives, eight physicians, three nurses, and three anesthetists.

Project design: The implementation team utilized a preand post-intervention design to evaluate the effectiveness of implementing the WHO SCC on the compliance of essential birth practices in the maternity service of Masaka District Hospital. The pre-intervention baseline assessment was done between January and February 2017 in order to measure the compliance of 29 essential birth practices. Data was collected through observation by the data collection team over 14 consecutive day shifts (7 am $-7 \mathrm{pm})$ and 4 night shifts (7 pm - $7 \mathrm{am})$. After the implementation of the WHO SCC, observations similar to the baseline assessment were repeated between March and April 2017. ${ }^{15}$

Engage: We involved the leadership of the hospital and maternity unit, and a quality improvement team for this SCC implementation was formed. The team included the researchers, the director, the clinical director, the head of maternity unit, the head of post-delivery unit, and staff representatives. The implementation team linked the SCC to the accreditation requirement as an incentive for the administration because higher accreditation scores resulted in higher reimbursement from the government. The team also received technical support from Ariadne Labs during the 2-day training on coaching. A multi-disciplinary team of 7 healthcare providers (4 midwives, 2 general practitioners, and 1 nurse) attended the training.

Launch: After engaging the administration and healthcare providers, the implementation team organized a 
1-hour Launch meeting with all maternity staff during a regular morning staff meeting to maximize attendance. During the Launch, the principal investigator provided a short presentation about the background, rationale, objectives, and implementation of the SCC project. He introduced the implementation team and their roles, and copies of the SCC were distributed to all participants in order to provide an initial training in small groups. Finally, the Hospital Director gave concluding remarks, encouraging all staff to use the SCC.

Support: During the implementation period in February 2017, Dr. Eugene Tuyishime provided a 2-day training session on the implementation of SCC to all clinical staff and administrators at the maternity unit. A trained multidisciplinary team of coaches was available to provide guidance and mentorship during the implementation. The coaches included 4 midwives, 2 general practitioners, and 1 anesthetist who received the coaching and implementation training. The coaches answered all concerns raised by the healthcare providers and the feedback was excellent. In addition, regular feedback was provided to the staff to ensure both timely positive reinforcement and corrective actions. The WHO SCC posters were posted on the walls around the delivery wards as reminders to clinicians of the essential birth practices and their importance.

Evaluation: A trained team of data collectors conducted direct observation of the 29 essential birth practices using the WHO SCC data collection tool at 4 pause points: 1) at admission (5 items), 2) before pushing and 1 minute after delivery (11 items), 3) within 1 hour post-delivery (6 items), and 4) within 1 hour before discharge (7 items). This team conducted four independent cross-sectional sampling of different women at the four pause points 2 weeks before and 2 weeks after the implementation of the checklist. The overall compliance rates of essential birth practices at the four pause points, and for each of the 29 practices, were compiled and compared before and after the intervention. The overall compliance of essential birth practices significantly increased from $46 \%$ pre-intervention to $56 \%$ post-intervention $(\mathrm{p}=0.005) .{ }^{15}$ Three pause points (pause points 2 , 3 , and 4) had shown improvement in compliance with essential birth practices, with the most improvement in pause point 4 (before discharge), which increased from $29 \%$ preintervention to $52 \%$ post-intervention $(\mathrm{p}=0.001) .{ }^{15}$

Challenges and lessons learned: The application of quality improvement principles, including engagement of leadership, strategic problem-solving skills, local ownership, coaching, and regular monitoring and evaluation, were critical to the success of SCC implementation. Challenges to implementation included increasing workloads for healthcare providers and adding a new form to the partograph. The SCC was not designed to address other factors leading to low quality of care, such as lack of equipment and supplies, insufficient team members, low salaries, and lack of continuous professional development.

\section{DISCUSSION}

\section{PROMISING PRACTICES FOR IMPLEMENTING THE WHO SAFE CHILDBIRTH CHECKLIST}

Through collaborative discussion regarding direct implementation experiences in India and Rwanda and findings from our surveys and interviews with SCC implementers around the world, we developed a list of promising practices according to each implementation phase (Figure 1). In the Project Design phase, we identified the importance of clarifying the purpose of the SCC and tailoring adaptations accordingly with attention to human-centered design principles. In the Engage phase, we identified the importance of securing buy-in from facility staff as well as leaders and demonstrating this support on an ongoing basis. In Rwanda, the SCC implementation was aligned with the accreditation requirement for quality improvement, which provided ongoing institutional support for the project. In the Launch phase, we identified the importance of the Launch event being both inclusive and inspiring. In Rwanda, all maternity staff were invited to the Launch event with the presence of the hospital director and other key members of the administration and quality improvement team. In the Support phase, we identified the importance of addressing bad habits that are difficult to change, infusing quality and safety culture into all workflows, strengthening the referral system, and stabilizing funding for the SCC implementation. In India, continuous coaching and recognition for positive performance on the SCC were critical for ongoing implementation. In the Evaluation phase, we identified the importance of analyzing data and disseminating findings on a regular basis to inform tailored improvements. In India, the team members used WhatsApp to troubleshoot SCC challenges and to provide accountability in monitoring and evaluation processes. These promising practices resonated across settings and the case studies provide more granularity about implementation approaches compared to previous studies. ${ }^{16}$

We acknowledge the limitations of developing promising practices from two case studies. However, we note the contextual differences in the implementation settings in India and Rwanda and were still able to identify common challenges and successes. Additionally, we incorporated lessons learned through our previous analysis of implementers around the world ${ }^{4}$ and discussions from our online community of practice to enhance generalizability to different settings. Given the additional detail provided through the two case studies, the promising practices may be particularly helpful for new implementers in guiding their approaches in addition to the high-level guidance in the WHO Safe Childbirth Checklist implementation guide. ${ }^{14}$

\section{CONCLUSIONS}

Our partnership with direct implementers of the SCC yielded important insights about how to adapt, implement, evaluate, and sustain use of the Checklist. Currently, there are few opportunities for SCC implementers to interact with and learn from each other. Our partnership is one model of sharing experiences and integrating lessons learned to 


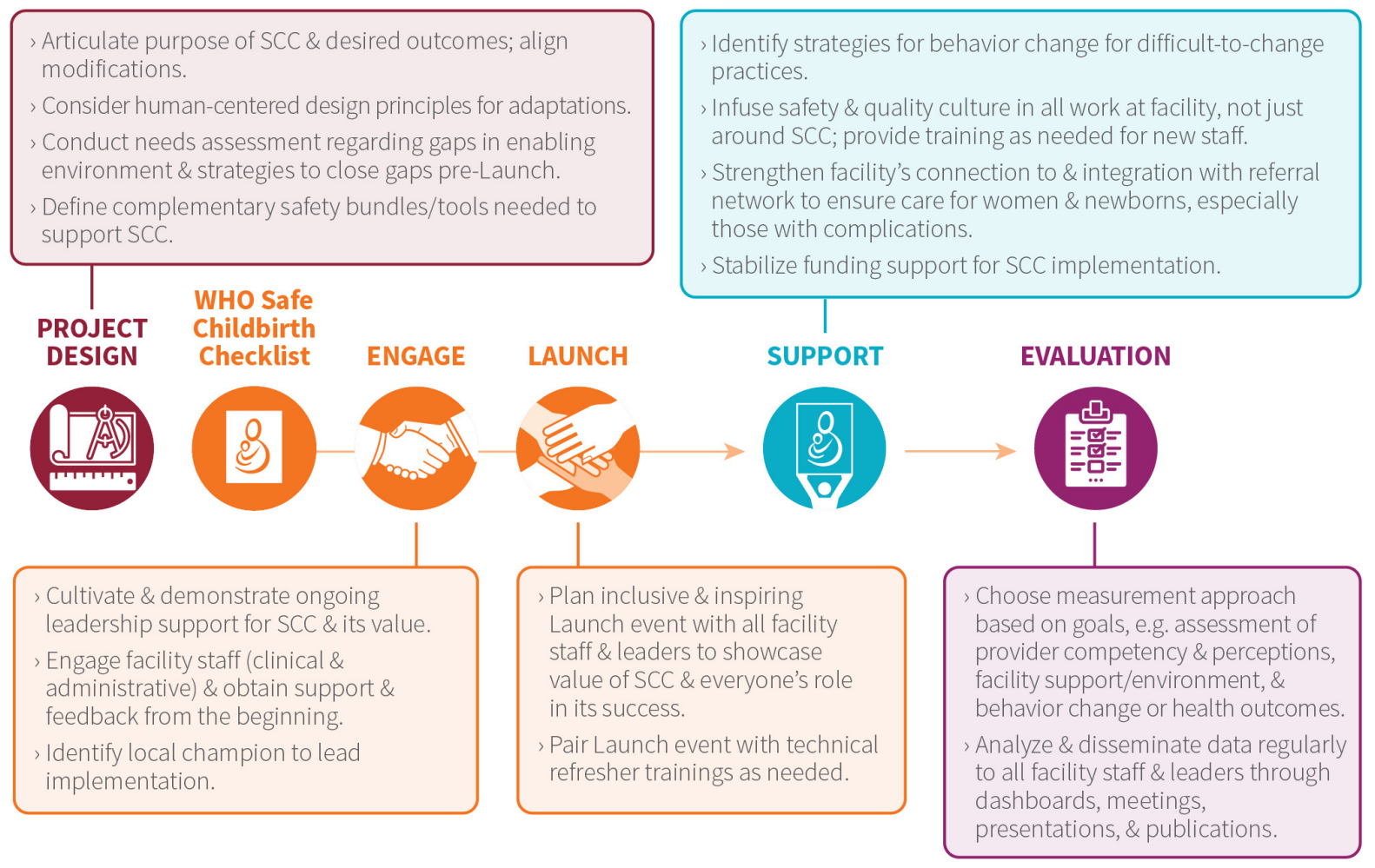

Figure 1. Promising practices for implementing the WHO Safe Childbirth Checklist.

share with a broader audience. Ariadne Labs is building a community of practice to facilitate similar connections. A common challenge we explored in this partnership was the lack of long-term funding to support ongoing refinement and continuous implementation of the Checklist. We call on funding agencies and donors to prioritize investment in SCC implementation, evaluation, and dissemination across settings. Through ongoing partnerships, we can build an evidence base for promising practices regarding SCC implementation around the world.

\section{FUNDING}

Ariadne Labs provided internal funding for this work.

\section{AUTHORSHIP CONTRIBUTIONS}

RLM - conception/design of the work; acquisition, analysis and interpretation of data; drafting the manuscript and revising the manuscript critically; AP - acquisition, analysis and interpretation of data; revising the manuscript critically; ET - acquisition, analysis and interpretation of data; revising the manuscript critically; LB - conception/design of the work; acquisition, analysis and interpretation of data; revising the manuscript critically; KEAS - conception/design of the work; acquisition, analysis and interpretation of data; revising the manuscript critically

\section{COMPETING INTERESTS}

The authors completed the Unified Competing Interest form at http://www.icmje.org/disclosure-of-interest/ (available upon request from the corresponding author), and declare no conflicts of interest.

\section{CORRESPONDENCE TO:}

Rose L. Molina, MD, MPH

Department of Obstetrics and Gynecology

Beth Israel Deaconess Medical Center

330 Brookline Ave, Kirstein 3

Boston, MA 02215.

617667-0887

rmolina@bidmc.harvard.edu

Submitted: November 02, 2021 GMT, Accepted: November 17, 2021 GMT 


\section{REFERENCES}

1. Spector JM, Lashoher A, Agrawal P, et al. Designing the WHO Safe Childbirth Checklist program to improve quality of care at childbirth. Int J Gynaecol Obstet. 2013;122(2):164-168. doi:10.1016/j.ijgo.201 $\underline{3.03 .022}$

2. Varghese B, Copas A, Kumari S, et al. Does the safe childbirth checklist (SCC) program save newborn lives? Evidence from a realistic quasi-experimental study, Rajasthan, India. Matern Health Neonatol Perinatol. 2019;5(3). doi:10.1186/s40748-019-0098-4

3. Walker D, Otieno P, Butrick E, et al. Effect of a quality improvement package for intrapartum and immediate newborn care on fresh stillbirth and neonatal mortality among preterm and lowbirthweight babies in Kenya and Uganda: a clusterrandomised facility-based trial. Lancet Glob Health. 2020;8(8):e1061-e1070. doi:10.1016/s2214-109x(20)3 $\underline{0232-1}$

4. Molina RL, Benski AC, Bobanski L, Tuller DE, Semrau KEA. Adaptation and implementation of the WHO Safe Childbirth Checklist around the world. Implement Sci Commun. 2021;2(1):76. doi:10.1186/s43 058-021-00176-Z

5. Praxedes A de O, Arrais L, de Araújo MAA, da Silva EMM, da Silva Gama ZA, de Freitas MR. Assessment of adherence to the Safe Childbirth Checklist in a public maternity hospital in Northeast Brazil. / Avaliação da adesão à Lista de Verificação de Segurança no Parto em uma maternidade pública no Nordeste do Brasil. Cadernos de Saúde Pública. 2017;33(10):e00034516-e00034516.

6. Carvalho ICB de M, de Souza Rosendo TMS, de Freitas MR, et al. Adaptation and validation of the World Health Organization's on Safe Childbirth Checklist for the Brazilian context. Revista Brasileira de Saúde Materno Infantil. 2018;18(2):401-436 (Pt). do $\mathrm{i}: 10.1590 / 1806-93042018000200009$

7. da Silva Gama ZA, Medeiros WR, SaturnoHernández PJ, et al. The WHO Safe Childbirth Checklist implementation: impact on the prescription of magnesium sulphate through a one-year longitudinal study. BMC Pregnancy Childbirth. 2020;20(1):154. doi:10.1186/s12884-020-2836-Z

8. Bitewulign B, Abdissa D, Mulissa Z, et al. Using the WHO Safe Childbirth Checklist to Improve Essential Care Delivery as Part of the District -Wide Maternal and Newborn Health Quality Improvement Initiative, a Time Series Study. Published online August 12, 2020. doi:10.21203/rs.3.rs-52505/v1
9. Abawollo HS, Tsegaye ZT, Desta BF, et al. Implementing a modified World Health Organization safe childbirth checklist in health centers of Ethiopia: a pre and post intervention study. BMC Pregnancy Childbirth. 2021;21(1):77. doi:10.1186/s12884-021-03 $\underline{565-3}$

10. Doria S, Diba F, Susanti SS, Vollmer S, Monfared IG. Mothers' experiences of quality of care and potential benefits of implementing the WHO safe childbirth checklist: a case study of Aceh Indonesia. BMC Pregnancy Childbirth. 2019;19(1):461. doi:10.118 6/s12884-019-2625-8

11. Albolino S, Dagliana G, Bellandi T, et al. Safety and quality of maternal and neonatal pathway: implementation of the modified WHO safe childbirth checklist in two hospitals of the tuscany center trust, italy. In: Bagnara S, Tartaglia R, Albolino S, Alexander T, Fujita Y, eds. Proceedings of the 20th Congress of the International Ergonomics Association (IEA 2018) Volume I: Healthcare Ergonomics. Vol 818. Advances in Intelligent Systems and Computing. Springer International Publishing; 2019:648-659. doi:10.1007/ 978-3-319-96098-2 80

12. Semrau KEA, Hirschhorn LR, Marx Delaney M, et al. Outcomes of a Coaching-Based WHO Safe Childbirth Checklist Program in India. N Engl J Med. 2017;377(24):2313-2324. doi:10.1056/nejmoa1701075

13. Litcanu M, Prostean O, Oros C, Mnerie AV. BrainWriting Vs. Brainstorming Case Study For Power Engineering Education. Procedia - Social and Behavioral Sciences. 2015;191:387-390. doi:10.1016/i.s bspro.2015.04.452

14. The World Health Organization. WHO Safe Childbirth Checklist Implementation Guide.; 2015. Accessed August 18, 2019. http://apps.who.int/iris/bit stream/10665/199177/1/9789241549455 eng.pdf?u a=1\&ua=1\%0Ahttp://apps.who.int/iris/bitstream/han dle/10665/199177/9789241549455 eng.pdf;jsessioni $\mathrm{d}=0$ A4AC889D1ADE8D54022250C34A271A1?sequenc $\underline{\mathrm{e}=1}$

15. Tuyishime E, Park PH, Rouleau D, Livingston $P$, Banguti PR, Wong R. Implementing the World Health Organization safe childbirth checklist in a district Hospital in Rwanda: a pre- and post-intervention study. Matern Health Neonatol Perinatol. 2018;4:7. do i:10.1186/s40748-018-0075-3

16. Perry W, Bagheri Nejad S, Tuomisto K, et al. Implementing the WHO Safe Childbirth Checklist: lessons from a global collaboration. BMJ Glob Health. 2017;2(3):e000241. doi:10.1136/bmigh-2016-000241 